\title{
ORIGINAL
}

\section{Mortality and host response aberrations associated with transient and persistent acute kidney injury in critically ill patients with sepsis: a prospective cohort study}

Fabrice Uhel ${ }^{1 *} \mathbb{D}$, Hessel Peters-Sengers ${ }^{1}$, Fahimeh Falahi ${ }^{1}$, Brendon P. Scicluna ${ }^{1,2}$, Lonneke A. van Vught ${ }^{1}$, Marc J. Bonten ${ }^{3,4}$, Olaf L. Cremer ${ }^{5}$, Marcus J. Schultz ${ }^{6,7,8}$ and Tom van der Poll ${ }^{1,9}$ on behalf of the MARS consortium

\begin{abstract}
Purpose: Sepsis is the most frequent cause of acute kidney injury (AKI). The "Acute Disease Quality Initiative Workgroup" recently proposed new definitions for AKI, classifying it as transient or persistent. We investigated the incidence, mortality, and host response aberrations associated with transient and persistent AKI in sepsis patients.

Methods: A total of 1545 patients admitted with sepsis to 2 intensive care units in the Netherlands were stratified according to the presence (defined by any urine or creatinine RIFLE criterion within the first $48 \mathrm{~h}$ ) and evolution of AKI (with persistent defined as remaining $>48 \mathrm{~h}$ ). We determined 30-day mortality by logistic regression adjusting for confounding variables and analyzed 16 plasma biomarkers reflecting pathways involved in sepsis pathogenesis $(n=$ 866) and blood leukocyte transcriptomes $(n=392)$.
\end{abstract}

Results: AKI occurred in $37.7 \%$ of patients, of which $18.4 \%$ was transient and $81.6 \%$ persistent. On admission, patients with persistent AKI had higher disease severity scores and more frequently had severe (injury or failure) RIFLE AKI stages than transient AKI patients. Persistent AKI, but not transient AKI, was associated with increased mortality by day 30 and up to 1 year. Persistent AKI was associated with enhanced and sustained inflammatory and procoagulant responses during the first 4 days, and a more severe loss of vascular integrity compared with transient AKI. Baseline blood gene expression showed minimal differences with respect to the presence or evolution of AKI.

Conclusion: Persistent AKI is independently associated with sepsis mortality, as well as with sustained inflammatory and procoagulant responses, and loss of vascular integrity as compared with transient AKI.

Keywords: Sepsis, Acute kidney injury, Host response, Mortality, Intensive care unit

*Correspondence: dr.f.uhel@gmail.com

${ }^{1}$ Center for Experimental and Molecular Medicine, Amsterdam

University Medical Centers, location Academic Medical Center, University

of Amsterdam, Room G2-130; Meibergdreef 9, 1105 AZ Amsterdam, The Netherlands

Full author information is available at the end of the article

\section{鱼




\section{Introduction}

Acute kidney injury (AKI) is a heterogeneous syndrome occurring in one out of two critically ill patients [1], associated with increased short- and long-term mortality [2], cardiovascular complications [3], and persistent renal dysfunction [4]. Sepsis is the main cause of AKI in intensive care units (ICUs), accounting for $50 \%$ of all cases $[2,5]$. The complex pathophysiology of sepsis and the high burden of AKI in this context $[6,7]$ led to define the simultaneous presence of both syndromes as sepsis-associated AKI (S-AKI) [8]. Except for renal replacement therapy $[9,10]$, no specific treatment exists to date.

Besides the severity of renal dysfunction [11], various patterns of renal recovery may impact the outcome of S-AKI [12]. Because the definition of recovery has been equivocal [13], the Acute Disease Quality Initiative Workgroup (ADQI) recently proposed to further classify AKI as "transient" or "persistent" according to a duration of less or more than 48 hours, respectively [14]. This definition is based on expert opinion and awaits clinical validation.

The singularity of S-AKI pathogenesis has been underlined by the description of normal to increased renal blood flow [15-18] and modest histopathological findings [19-22], contrasting with the long-standing theory of renal hypoperfusion-induced acute tubular necrosis [23]. Experimental studies suggest a role for oxidative stress, inflammation [24], microvascular shunting [25], and tubular cell stress [26], but data in patients are scarce and the influence of the unbalanced sepsis host response on recovery from S-AKI is unknown.

The primary objective of this study was to determine the incidence and mortality associated with transient and persistent AKI in critically ill patients admitted with sepsis using the new ADQI definitions [14]. Additionally, we sought to determine differences in the host response to the inciting sepsis event between patients without AKI, and those with transient or persistent AKI by analysis of plasma biomarkers implicated in the pathogenesis of critical illness and whole-genome blood leukocyte transcriptomes.

This work was partially presented at the French Intensive Care Society international Congress on February 5-7, 2020, in Paris [27].

\section{Take-home message}

Persistent but not transient AKI (as defined according to the new "Acute Disease Quality Initiative Workgroup" definitions) independently contributes to short- and long-term mortality during sepsis. Compared to transient AKI, persistent AKI is associated with sustained systemic inflammation, coagulation activation and loss of vascular integrity.

\section{Methods}

\section{Study design and population}

This study was part of the Molecular Diagnosis and Risk Stratification of Sepsis (MARS) project, a prospective observational study conducted between January 2011 and December 2013 in the mixed ICUs of two tertiary teaching hospitals in the Netherlands (Academic Medical Center, Amsterdam and University Medical Center Utrecht, Utrecht; ClinicalTrials.gov identifier NCT01905033) $[28,29]$.

For the current analysis, all consecutive patients with sepsis older than 18 years of age and with an expected length of stay greater than $24 \mathrm{~h}$ were included via an optout consent method approved by the institutional review boards of both hospitals (IRB No. 10-056C). The plausibility of an infection was assessed daily using a four-point scale (ascending from none, possible, probable, to definite) [28]. Readmissions and patients transferred from another ICU were excluded, except when patients were referred to one of the study centers the same day of presentation to the first ICU.

In additional analyses, patients with sepsis were compared with patients admitted to the ICU for a noninfectious condition originally selected as control groups for patients with pneumonia, abdominal sepsis, or septic shock [30-32].

\section{Clinical variables and definitions}

Sepsis was defined according to the new definition as the presence of an infection (with a likelihood of at least possible) diagnosed within 24 hours after ICU admission and organ dysfunction(s) represented by two or more Sequential Organ Failure Assessment (SOFA) points [33]. The presence of AKI was assessed prospectively and daily, using the risk, injury, failure, loss, and end-stage kidney disease (RIFLE) classification [34] based on maximum severity by either creatinine or urine output criteria. This definition used at the time of the study is concordant with the more recent Kidney Disease: Improving Global Outcomes (KDIGO) definition [35, 36] (Online resource eTable 1). When a recent reference creatinine value was not available, baseline creatinine was estimated using the 
MDRD equation by back calculation from an estimated glomerular filtration rate of $75 \mathrm{~mL} / \mathrm{min} / 1.73 \mathrm{~m}^{2}$ [37].

AKI was defined as the presence of at least one criterion consistent with the risk (R), injury (I), or failure (F) stage within the first 48 hours after ICU admission; otherwise patients were classified as having no AKI. In accordance with recent expert consensus [14], AKI was retrospectively classified as "persistent" if one or more RIFLE criteria remained present beyond $48 \mathrm{~h}$ from AKI onset, or if they normalized within $48 \mathrm{~h}$ but relapsed within the next $48 \mathrm{~h}$. Reversal of RIFLE criteria within $48 \mathrm{~h}$ of AKI onset and for at least $48 \mathrm{~h}$ defined AKI as "transient". When 48-h follow-up was not possible due to death or discharge from ICU, AKI was classified as persistent if patients met one of the RIFLE criteria for their last measurement, otherwise as transient [14].

For other definitions, see Online resource eMethods.

\section{Biomarker assays and whole blood gene expression analyses}

See online resource eMethods.

\section{Statistical analyses}

Baseline characteristics were compared with ANOVA for normally distributed and Kruskal-Wallis test for nonnormally distributed continuous variables. Categorical data were analyzed using Chi-square or Fisher's exact test. Biomarker trajectories over time were analyzed using a general mixed model analysis in which a linear regression model was fitted on logarithmically transformed data, taking group (no AKI, transient AKI, persistent AKI), time, and their interaction as fixed effects, and patient-specific intercept and slope of time as random effects. Differences in biomarker distribution between groups at each time point were compared using KruskalWallis tests followed by Dunn's post hoc tests.

To investigate the independent association between the presence and the evolution of AKI and mortality, we performed a logistic regression described in the online resource.

Receiver operating characteristic analyses, the handling of missing data, and sensitivity and subgroup analyses are described in the online resource.

Data analyses were performed in R (v 3.5.1, R Foundation for Statistical Computing, Vienna, Austria). Bonferroni multiple-comparison-adjusted $P$ values less than .05 defined significance. Benjamini-Hochberg-adjusted $P$ values defined significant differential gene expression and pathway enrichment.

\section{Results}

\section{Patient characteristics}

During the 3-year study period, 2787 consecutive ICU admissions for sepsis were included. A total of 1242 admissions were excluded, involving readmissions, transfers from other ICUs, patients with prior chronic kidney disease, or patients in whom the RIFLE score within the first 24 hours of ICU admission was not available (Online resource eFigure 1), resulting in a final study cohort of 1545 patients admitted with sepsis. Of these, 968 (62.7\%) did not have AKI, while 577 (37.3\%) were diagnosed with AKI within the first $48 \mathrm{~h}$ of admission (Table 1). Of patients presenting with AKI, in 106 (18.4\%) renal function recovered within $48 \mathrm{~h}$ of onset and for at least $48 \mathrm{~h}$ (transient AKI), and 471 (81.6\%) had persistent AKI. Small differences were observed across study centers, likely related to differences in clinical presentation (Online resource, eTable 2).

Compared with patients without AKI, patients with AKI differed minimally in demographics, chronic comorbidities, or medications (Table 1). Patients with persistent AKI were older, more often received diuretic therapy, and had a higher Charlson comorbidity index. Both transient and persistent AKI were strongly associated with the severity of disease, as reflected by higher APACHE IV scores, higher modified total and non-renal (m)SOFA scores, a more frequent occurrence of shock, ARDS, and requirement for inotropes and higher doses of vasopressors, when compared with absence of AKI (Table 1). Exposure to nephrotoxic drugs was also more frequent in these patients (Table 1).

Abdominal, cardiovascular, urinary tract, and skin or soft tissue infections were more prevalent in patients with persistent AKI (Table 1), possibly related to a more severe disease presentation and more frequent use of nephrotoxic drugs (data not shown). Gram-negative bacteria and yeasts were more frequently identified in this group (Online resource eTable 3).

\section{Severity and evolution of AKI}

Persistent AKI was associated with more severe renal dysfunction at baseline, as reflected by higher urea and creatinine concentrations and a lower urine output compared with transient AKI (Table 1). 33 (31.3\%) of patients with a persistent AKI had mild (none or R) RIFLE criteria, whereas 149 (31.1\%) of patients with a transient AKI fulfilled severe (I or F) criteria at the time of admission (Fig. 1a-e). Median creatinine levels stayed within the normal range in patients without AKI on admission, as well as in patients with transient AKI after the first $48 \mathrm{~h}$. In patients with persistent $\mathrm{AKI}$, median creatinine levels remained elevated for up to 28 days (Fig. 1f). 
Table 1 Baseline characteristics and outcomes of patients admitted to the ICU with sepsis, stratified according to the presence and evolution of acute kidney injury

\begin{tabular}{|c|c|c|c|c|}
\hline & \multirow[t]{2}{*}{ No AKI $(n=968)$} & \multicolumn{2}{|l|}{ AKI } & \multirow[t]{2}{*}{$P$ value } \\
\hline & & Transient AKI $(n=106)$ & Persistent AKI $(n=471)$ & \\
\hline \multicolumn{5}{|l|}{ Demographics } \\
\hline Age, years & $62[48-71]$ & $63[49-72]$ & $65[54-73]^{*}$ & $<.001$ \\
\hline Male sex & $606(62.6)$ & $61(57.5)$ & $275(58.4)$ & .23 \\
\hline Race, white & $847(88)$ & $90(84.9)$ & $419(90.3)$ & .21 \\
\hline Medical admission & $721(74.5)$ & $76(71.7)$ & $340(72.2)$ & .57 \\
\hline \multicolumn{5}{|l|}{ Chronic comorbidities } \\
\hline None & $318(32.9)$ & $31(29.2)$ & $135(28.7)$ & .25 \\
\hline Cardiovascular compromise & $229(23.7)$ & $34(32.1)$ & $127(27)$ & .10 \\
\hline Hypertension & $229(23.7)$ & $35(33.0)$ & $134(28.5)$ & .030 \\
\hline Diabetes & $159(16.4)$ & $20(18.9)$ & $92(19.5)$ & .31 \\
\hline Liver cirrhosis & $13(1.3)$ & $4(3.8)$ & $15(3.2)$ & .021 \\
\hline Immune compromise & $184(19)$ & $18(17)$ & $94(20)$ & .79 \\
\hline Malignancy & $200(20.7)$ & $20(18.9)$ & $114(24.2)$ & .25 \\
\hline Charlson comorbidity index & $3[1-5]$ & $3[2-5]$ & $3[2-5]^{*}$ & $<.001$ \\
\hline \multicolumn{5}{|l|}{ Chronic medication } \\
\hline Diuretics & $185(19.1)$ & $26(24.5)$ & $120(25.5)^{*}$ & .016 \\
\hline ACE inhibitors/ARBs & $223(23)$ & $24(22.6)$ & $128(27.2)$ & .21 \\
\hline Calcium-entry blockers & $122(12.6)$ & $18(17)$ & $68(14.4)$ & .33 \\
\hline Beta-adrenergic blockers & $222(22.9)$ & $28(26.4)$ & $135(28.7)$ & .06 \\
\hline NSAIDs and Cox II inhibitors & $112(11.6)$ & $13(12.3)$ & $52(11)$ & .90 \\
\hline Oral antidiabetic drugs & $113(11.7)$ & $15(14.2)$ & $67(14.2)$ & .33 \\
\hline Corticosteroids & $99(10.2)$ & $9(8.5)$ & $47(10)$ & .90 \\
\hline Antiplatelet drugs & $208(22.5)$ & $24(23.3)$ & $112(24.5)$ & .71 \\
\hline \multicolumn{5}{|c|}{ Severity at time of admission to ICU } \\
\hline APACHE IV score & $67[52-85]$ & $75[66-94]^{*}$ & $95[74-118]^{*+}$ & $<.001$ \\
\hline Acute physiology score & $55[42-71]$ & $64[54-80]^{*}$ & $81[62-105]^{*+}$ & $<.001$ \\
\hline mSOFA score & $5[3-7]$ & $8[6-9]^{*}$ & $9[7-12]^{*+}$ & $<.001$ \\
\hline Non-renal mSOFA score & $5[3-7]$ & $7[5-8]^{*}$ & $8[6-9]^{*+}$ & $<.001$ \\
\hline Shock & $362(37.4)$ & $73(68.9)^{*}$ & $349(74.1)^{*}$ & $<.001$ \\
\hline ARDS & $192(19.8)$ & $30(28.3)$ & $135(28.7)^{*}$ & $<.001$ \\
\hline \multicolumn{5}{|l|}{ Therapy during the first $24 \mathrm{~h}$} \\
\hline Mechanical ventilation & $773(79.9)$ & $89(84)$ & $396(84.1)$ & .13 \\
\hline Vasopressors & $503(52)$ & $83(78.3)^{*}$ & $393(83.4)^{*}$ & $<.001$ \\
\hline Dose of vasopressors (mg) ${ }^{\mathrm{a}}$ & $5.8[2.0-13.3]$ & $11.1[4.9-21.8]^{*}$ & $15.1[5.6-33.5]^{*}$ & $<.001$ \\
\hline Inotropes & $40(4.1)$ & $10(9.4)^{*}$ & $82(17.4)^{*+}$ & $<.001$ \\
\hline Dose of inotropes $(\mathrm{mg})^{a}$ & $151.3[46.7-254.7]$ & $126.4[57.5-303.8]$ & $179.4[63.2-312.7]$ & .52 \\
\hline RRT & $6(0.6)$ & $3(2.8)^{*}$ & $98(20.9)^{*}+$ & $<.001$ \\
\hline Nephrotoxic drugs ( $\geq 1$ ) & $379(39.2)$ & $57(53.8)^{*}$ & $280(59.4)^{*}$ & $<.001$ \\
\hline Aminoglycoside & $128(13.2)$ & $24(22.6)^{*}$ & $129(27.4)^{*}$ & $<.001$ \\
\hline Glycopeptide & $106(11)$ & $12(11.3)$ & $78(16.6)^{*}$ & .012 \\
\hline Colloid & $163(16.8)$ & $30(28.3)^{*}$ & $175(37.2)^{*}$ & $<.001$ \\
\hline Other $^{b}$ & $94(9.7)$ & $10(9.4)$ & $37(7.9)$ & .53 \\
\hline \multicolumn{5}{|l|}{ Source of infection } \\
\hline Pulmonary tract & $562(58.1)$ & $54(50.9)$ & $164(34.8)^{*+}$ & $<.001$ \\
\hline Abdominal & $134(13.8)$ & $27(25.5)^{*}$ & $129(27.4)^{*}$ & $<.001$ \\
\hline Cardiovascular & $70(7.2)$ & $6(5.7)$ & $64(13.6)^{*}$ & $<.001$ \\
\hline Urinary tract & $43(4.4)$ & $6(5.7)$ & $44(9.3)^{*}$ & .001 \\
\hline
\end{tabular}


Table 1 (continued)

\begin{tabular}{|c|c|c|c|c|}
\hline & \multirow[t]{2}{*}{ No AKI $(n=968)$} & \multicolumn{2}{|l|}{ AKI } & \multirow[t]{2}{*}{$P$ value } \\
\hline & & Transient AKI $(n=106)$ & Persistent AKI $(n=471)$ & \\
\hline CNS & $56(5.8)$ & $3(2.8)$ & $12(2.5)^{*}$ & .013 \\
\hline Skin or soft tissue & $22(2.3)$ & $4(3.8)$ & $26(5.5)^{*}$ & .006 \\
\hline Other $^{c}$ & $78(8.1)$ & $6(5.7)$ & $20(4.2)^{*}$ & .021 \\
\hline Unknown & $3(0.3)$ & $0(0)$ & $12(2.5)^{*}$ & $<.001$ \\
\hline \multicolumn{5}{|c|}{ Renal function during the first $24 \mathrm{~h}$} \\
\hline Creatinine $(\mu \mathrm{mol} / \mathrm{L})$ & $79[60-102]$ & $136[93-170]^{*}$ & $175[131-246]^{*}$ & $<.001$ \\
\hline Urea (mmol/L) & $6.7[4.6-9.9]$ & $11.4[8.3-17.2]^{*}$ & $13.2[9.4-19.1]^{*}$ & $<.001$ \\
\hline Bicarbonate, minimal (mmol/L) & $22.3[19.1-25.9]$ & $18.9[16.9-22.8]^{*}$ & $16.2[13.2-20.3]^{*+}$ & $<.001$ \\
\hline Urine output $(\mathrm{mL})$ & 1900 [1303-2815] & $1405[1015-2440]^{*}$ & $940[408-1643]^{*+}$ & $<.001$ \\
\hline \multicolumn{5}{|l|}{ Outcome } \\
\hline Duration of initial MV (days) & $2[1-5]$ & $3[1-7]^{*}$ & $2[1-8]^{*}$ & $<.001$ \\
\hline Recurrence of MV & $24(2.5)$ & $9(8.5)^{*}$ & $24(5.1)^{*}$ & .001 \\
\hline MV-free days ${ }^{d}$ & 86 [46-89] & 84 [30-88] & $40[1-86]^{*+}$ & $<.001$ \\
\hline Use of RRT & $25(2.6)$ & $6(5.7)^{*}$ & $162(34.4)^{*}$ & $<.001$ \\
\hline RRT-free days ${ }^{\mathrm{d}}$ & 90 [59-90] & 90 [42-90] & $55[3-90]^{*}$ & $<.001$ \\
\hline \multicolumn{5}{|l|}{ Complications $^{e}$} \\
\hline None & $870(89.9)$ & $92(86.8)$ & $403(85.6)$ & .048 \\
\hline ICU-acquired AKI & $57(5.9)$ & $4(3.8)$ & $12(2.5)^{*}$ & .014 \\
\hline ICU-acquired ARDS & $20(2.1)$ & $3(2.8)$ & $10(2.1)$ & .81 \\
\hline ICU-acquired infection & $47(4.9)$ & $10(9.4)^{*}$ & $56(11.9)^{*}$ & $<.001$ \\
\hline ICU length of stay (days) & $4[2-8]$ & $6[4-9]^{*}$ & $5[3-11]^{*+}$ & $<.001$ \\
\hline Hospital length of stay (days) & 16 [8-29] & 18 [10-33] & $15[5-34]$ & .05 \\
\hline ICU-mortality & $109(11.3)$ & $13(12.3)$ & $178(37.8)^{*+}$ & $<.001$ \\
\hline 30-day mortality & $197(20.4)$ & $21(19.8)$ & $203(43.1)^{*+}$ & $<.001$ \\
\hline 60-day mortality & $245(25.3)$ & $29(27.4)$ & $232(49.3)^{*}$ & $<.001$ \\
\hline 90-day mortality & $274(28.3)$ & $31(29.2)$ & $247(52.4)^{*}$ & $<.001$ \\
\hline 1-year mortality & $365(37.7)$ & $40(37.7)$ & $278(59)^{*+}$ & $<.001$ \\
\hline ICU-free days $^{d}$ & $84[45-87]$ & 82 [27-86] & $47[0-84]^{*}+$ & $<.001$ \\
\hline
\end{tabular}

$A C E$ angiotensin-converting enzyme, $A K I$ acute kidney injury, $A P A C H E$ acute physiology and chronic health evaluation, $A R D S$ acute respiratory distress syndrome, $A R B S$ angiotensin II receptor blockers, CNS central nervous system, ICU intensive care unit, MV mechanical ventilation, NSAIDs non-steroidal anti-inflammatory drugs, RRT renal replacement therapy, mSOFA modified sequential organ failure assessment (excluding central nervous system component).

Data presented as median [interquartile range], or $n$ (\%). Continuous variables were compared using the Kruskal-Wallis test. Associations between categorical variables were tested using the Fisher's exact test. $P$ value represents comparisons between the three groups.

* Significant versus no AKI, using a Dunn's test of multiple comparisons using rank sums (continuous variables) or a pairwise test for a multi-level 2-dimensional matrix (categorical variables).

† Significant versus transient AKI, using a Dunn's test of multiple comparisons using rank sums (continuous variables) or a pairwise test for a multi-level twodimensional matrix (categorical variables).

a Cumulative dose given for patients who received vasopressors (epinephrine, norepinephrine or dopamine, expressed in norepinephrine-equivalent dose) or dobutamine during the first $24 \mathrm{~h}$

b Other nephrotoxic drug; includes any of the following medications: nonsteroidal anti-inflammatory drugs, angiotensin-converting enzyme inhibitors, angiotensin II receptor blockers, amphotericin $\mathrm{B}$, acyclovir, foscarnet, calcineurin inhibitors.

c Other sites of infection: infections of bones and joints $(n=19)$, oral infections $(n=8)$, postoperative wound infections ( $n=20)$, upper respiratory tract infections ( $n$

$=20$ ), viral systemic infections $(n=6)$, endometritis $(n=4)$, other $(n=27)$.

d Between inclusion and day 90 .

e Complications were defined as ICU-acquired when diagnosed more than $48 \mathrm{~h}$ after admission to the ICU.

\section{Outcome}

Compared with patients without AKI, patients with transient or persistent AKI had a longer ICU length of stay and were more prone to develop an ICU-acquired infection (Table 1). Patients with persistent AKI had a reduced number of days alive and free from mechanical ventilation, free from renal replacement therapy, or out of the ICU. Mortality at day 30 increased along with AKI severity on admission (Online resource eFigure 2). In addition, patients with persistent AKI, but not with transient AKI 
showed increased mortality in the ICU and up to one year after admission (Table 1, Online resource eFigure 3).

In a logistic regression analysis, persistent but not transient AKI was associated with an increased crude risk of mortality by day 30 and up to 1 year compared with no AKI (Table 2, Online resource eTable 4). After adjustment for severity and other confounding factors, the specific association between the persistence of AKI and 30 -day mortality (OR 2.42, 95\% CI 1.28- 4.58; $P=.006$, Table 2) and 1-year mortality (OR 2.10, 95\% CI $1.12-$ 3.92; $P=.020$, Online resource eTable 4 ) remained. The Hosmer-Lemeshow test showed proper model calibration (Online resource eFigure 4).

\section{Plasma renal function and host response biomarkers}

Biomarkers indicative of renal function and host response pathways implicated in sepsis pathogenesis were measured on admission to the ICU and at day 2 and day 4 thereafter in the subset of sepsis patients with a definite or probable infection likelihood enrolled during the first 2.5 years $(n=866,56 \%)$. This subgroup was comparable to the whole cohort (Online resource eTable 5).

The plasma levels of cystatin $C$ and neutrophil gelatinase-associated lipocalin (NGAL) have been used as markers of renal function [38-40]. Both cystatin C and NGAL were elevated in patients with sepsis compared with healthy volunteers irrespective of the presence of AKI, and further increased in patients with AKI compared with patients without AKI (Online resource eFigure 5). On admission, cystatin $C$ but not NGAL concentrations were significantly higher in patients who went on to have a persistent AKI compared with those in whom AKI was transient $(P<.001)$.

Patients with sepsis showed typical features of a dysregulated host response [41, 42] (Fig. 2). Compared with patients without renal dysfunction, patients with transient and persistent AKI had higher levels of interleukin (IL)-6, IL-8, IL-10, and matrix metalloproteinase- 8 on admission. Patients with persistent AKI, but not with transient $\mathrm{AKI}$, also had more prominent signs of endothelial cell activation (elevated plasma levels of fractalkine, soluble E-selectin, soluble intercellular adhesion molecule-1), more severe loss of vascular integrity (increased angiopoietin-2/angiopoietin-1 ratio), and stronger activation of the coagulation system (prolonged prothrombin time and activated partial thromboplastin time, elevated plasma levels of D-dimer, and reduced platelet counts and levels of natural anticoagulants antithrombin and protein $\mathrm{C}$ ); these aberrations persisted up until day 4 (Fig. 2, Online resource, eTable 6). Similarly, blood leukocytes were higher in patients with transient and persistent AKI (Online resource eFigure 6). Differences at admission, albeit significant, did not make these biomarkers individually suitable for the early prediction of evolution towards persistent AKI (Online resources, eTable 7).

\section{Whole blood leukocyte transcriptome analysis}

In the subgroup of sepsis patients with an infection likelihood of definite or probable enrolled during the first 1.5 years of the study period, blood leukocyte genomewide RNA profiles were determined on admission $(n=$ 392, of whom 225 without AKI, 36 with transient AKI and 131 with persistent AKI) (Online resource eTable 8). Blood RNA profiles of patients were initially compared to those of 42 healthy controls. Both patients without and with AKI displayed strong blood transcriptome alterations, encompassing $71-80 \%$ of all genes present on the array (Fig. 3a). Of the altered RNA transcripts, 79\% were common to patients without AKI and those with transient or persistent AKI (Fig. 3b) and this common response showed strongly correlated gene expression fold changes (Fig. 3c). Consistent with earlier studies in sepsis patients $[29,43]$, pathway analysis of the common response revealed a typical overexpression of genes involved in both pro- and anti-inflammatory innate immune responses and metabolic pathways, and a concomitant underexpression of genes of lymphocyte and antigen presentation pathways (Online resource eFigure 7). Direct comparison of transcriptomes between

\footnotetext{
(See figure on next page.)

Fig. 1 Evolution of acute kidney injury in patients with sepsis. a Chord diagrams depicting the relationship between the severity of AKI upon ICUadmission and the subsequent presence and evolution of AKI over the first 48 hours. The bottom part of the diagram represents patients ranked by initial RIFLE score calculated upon ICU admission, and the top part represents the same patients ranked according to the presence and evolution of AKI over the first $48 \mathrm{~h}$ (no AKI, transient or persistent AKI). Ribbons show for every patient the connection between the initial RIFLE score and the subsequent evolution of AKI. The first diagram (a) represents all patients combined. The diagrams below (b-e), highlight each individual RIFLE severity score separately. Admission RIFLE score was "none" for all $(n=968)$ patients without AKI. Of patients with transient AKI, 13 (12.3\%), 60 (56.6), 24 (22.6), and 9 (8.5\%) had an admission RIFLE score of "none," "at risk", "injury," or "failure," respectively. Of patients who developed persistent AKI, 39 (8.3\%), 110 (23.4\%), 147 (31.2\%), and 175 (37.2\%) had an admission RIFLE score of "none", "at risk", "injury", or "failure", respectively. f Plasma creatinine over time stratified according to the evolution of AKI after admission to the intensive care unit (ICU). Data are presented as mean and standard error of the mean. Numbers below axes indicate the number of patients still present in the ICU for each group. Note: mean creatinine levels showed small increases at day 8 and day 20 in the transient AKI group due to the occurrence of ICU-acquired AKI in 4 patients in whom renal function initially recovered after the initial episode of AKI
} 


\section{a All admission RIFLE scores combined}

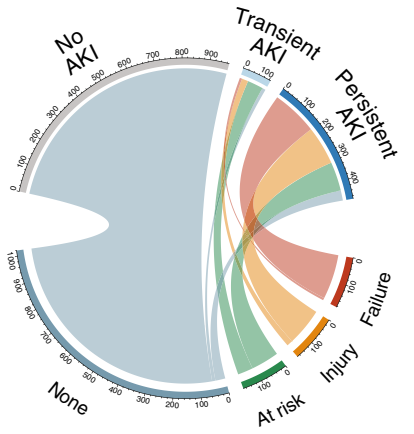

b Admission RIFLE "None"

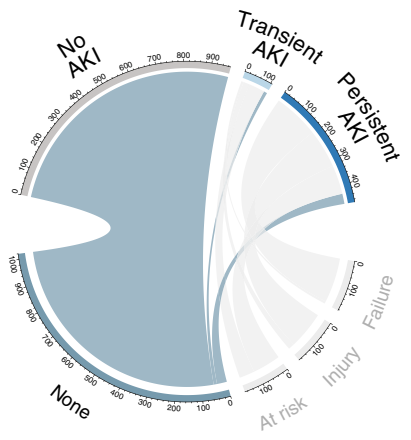

d Admission RIFLE "Injury"

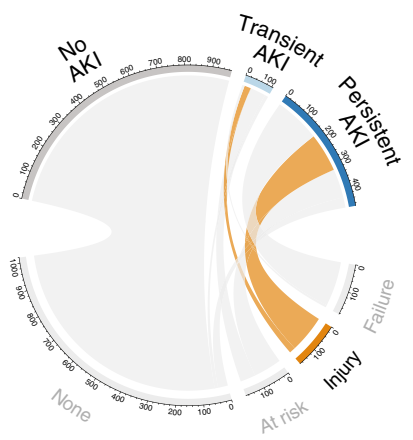

C Admission RIFLE "At risk"

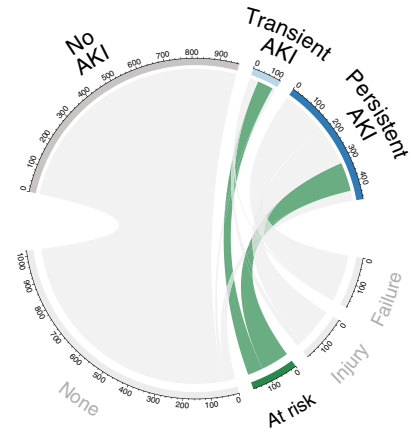

e Admission RIFLE "Failure"

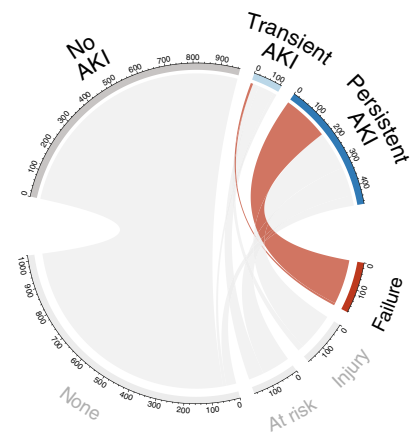

f

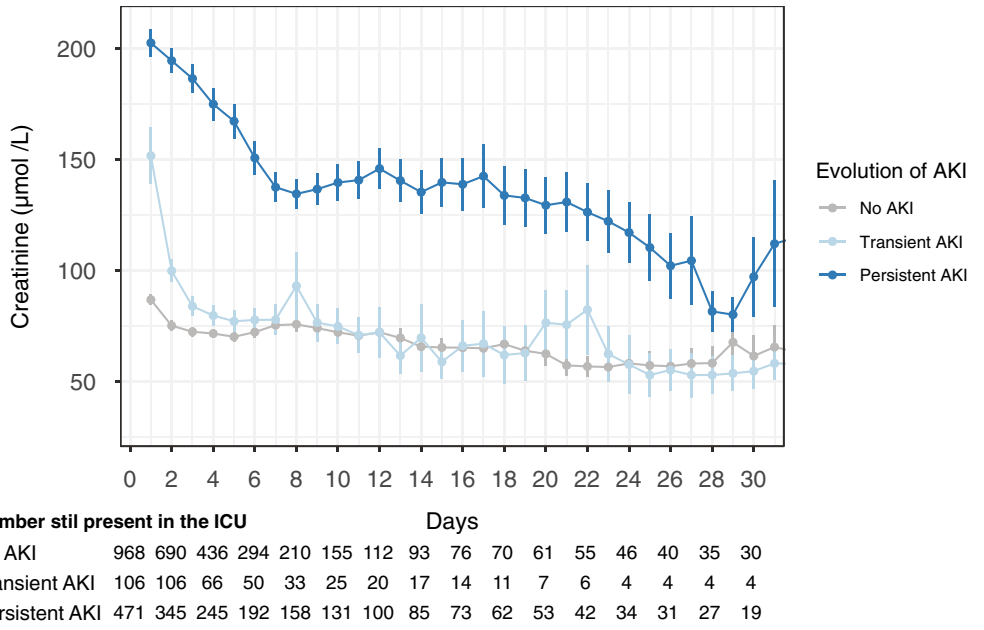


Table 2 Logistic regression analysis evaluating the influence of the evolution of acute kidney injury on 30-day mortality

\begin{tabular}{|c|c|c|c|c|}
\hline & $\begin{array}{l}\text { Odds ratio }(95 \% \\
\mathrm{Cl})\end{array}$ & $P$ value & Wald test $\chi^{2}$ (df) & $P$ \\
\hline \multicolumn{5}{|l|}{ Crude model $^{\mathrm{a}}$} \\
\hline No AKI & 1 (reference) & - & $82.4(2)$ & $P<.001$ \\
\hline Transient AKI & $0.97(0.58-1.6)$ & .90 & & \\
\hline Persistent AKI & $2.96(2.33-3.77)$ & $<.001$ & & \\
\hline
\end{tabular}

\section{Adjusted model $^{b}$}

$\begin{array}{lllll}\text { No AKI } & 1 \text { (reference) } & - & 10.5(2) & P=.005 \\ \text { Transient AKI } & 1.25(0.58-2.7) & .56 & & \\ \text { Persistent AKI } & 2.42(1.28-4.58) & .006 & & \end{array}$

$A K l$ acute kidney injury, $\mathrm{Cl}$ confidence interval, $d f$ degrees of freedom.

a Unadjusted model.

b Adjusted for age, admission RIFLE score, APACHE acute physiology score, source of infection, and modified Charlson comorbidity index (omitting the age parameter).

patients without, transient, or persistent AKI showed no or minimal statistically significant differences (Fig. 3d). Only the comparison of persistent to no AKI revealed a global decrease in expression of genes involved in key innate and adaptive immune cell functions, concomitant with increased expression of specific metabolic pathway genes (Online resource eFigure 8).

\section{Subgroup and sensitivity analyses}

Multiple subgroup and sensitivity analyses were performed to assess the robustness of the analyses presented above (Online resources, eResults).

These analyses focused on patients still present in the ICU on day 4 (Online resource eTables 10, 11, eFigure 9), patients matched for severity and baseline differences (Online resource eTables 12,13), patients with shock (Online resource eTables 14-24, eFigures 10-13), and patients with severe (RIFLE I-F) AKI (Online resources eTables 25-28, eFigure 14).

Additionally, we investigated the impact of alternative cutoffs to define persistent AKI (Online resource eTables 29-36, eFigures 15-16) and described a group of patients with noninfectious admission diagnoses (Online resource eTables 37-40, eFigures 17-19)

These additional analyses largely confirmed the findings in the primary cohort.

\section{Discussion}

In this prospectively enrolled cohort of critically ill patients with sepsis, we used the new ADQI consensus definitions of transient and persistent AKI [14] to determine the incidence of distinct AKI trajectories and the mortality associated with the persistence of AKI. More than a third of sepsis patients had AKI upon ICU admission, of which the majority was severe (RIFLE stage I or F), and persistent in 4 out of every 5 patients. In contrast to transient AKI, persistent AKI independently impacted patient outcome, with more than twofold increased odds for 30-day mortality when compared with no AKI. This study further suggests that besides the severity of AKI, the duration of renal dysfunction independently affects short-term and up to 1-year patient survival. Aside from the sepsis-related sustained disturbance of homeostasis [44], the persistence of AKI may specifically impact patient survival through prolonged exposure to harmful adverse events, such as fluid overload, electrolyte and acid-base disturbances, cardiovascular events [3], and the development of chronic kidney disease [45, 46]. Our data are in agreement with findings of previous investigations based on retrospective calculation of AKI severity scores $[3,47,48]$, using different definitions for renal recovery ranging from $24 \mathrm{~h}$ to hospital discharge [7, 12, 47-51], or using ICU or hospital mortality as primary outcomes [47-49].

The various patterns of renal recovery after AKI thus far have been mainly attributed to the severity of the underlying disease or of the AKI itself [13]. Except knowledge derived from experimental studies and postmortem observations, little is known about the pathophysiological processes responsible for the onset and persistence of kidney dysfunction during sepsis. In a subgroup of patients, we measured 16 host response biomarkers reflective of important pathways involved in the pathogenesis of sepsis, during the first 4 days after ICU admission. The presence of AKI was associated with enhanced

\footnotetext{
(See figure on next page.)

Fig. 2 Host response biomarkers in patients with sepsis during the first 4 days of ICU stay stratified according to the evolution of acute kidney injury after admission. Biological parameters are classified as $\mathbf{a}$ inflammatory responses, $\mathbf{b}$ endothelial cell activation, and $\mathbf{c}$ coagulation activation biomarkers. Data are presented as box and whiskers, as specified by Tukey. Dotted lines represent median values obtained in 27 healthy agematched healthy subjects. Overall $P$ values were derived from the linear mixed model in which the group, or the interaction of time $x$ group (i.e. the trajectory) were defined as fixed effects, and patient-specific intercept and slopes were defined as random effects. Comparisons between groups at specific days were performed using the Kruskal-Wallis test followed by Dunn's post hoc tests of multiple comparisons using rank sums. ${ }^{*} P<.05$, ${ }^{* *} P$ $<.01,{ }^{* * *} P<.001,{ }^{* * * *} P<.0001$. AKI, acute kidney injury; ANG, angiopoietin; aPTT, activated partial thromboplastin time; IL, interleukin; MMP, matrix metalloproteinase; PT, prothrombin time; sE-Selectin, soluble E-selectin; sICAM, soluble intercellular adhesion molecule
} 


\section{a Inflammatory responses}
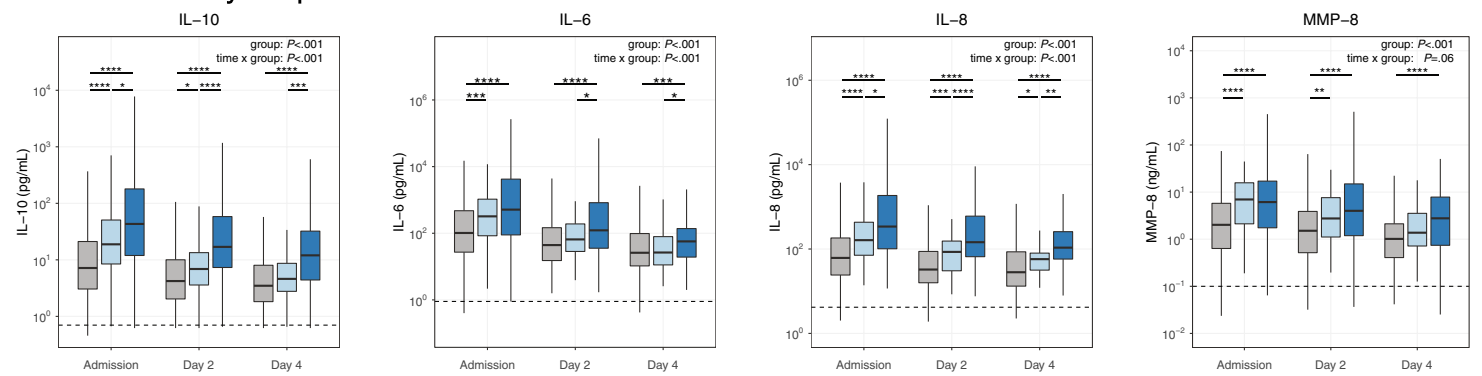

\section{b Endothelial cell activation}
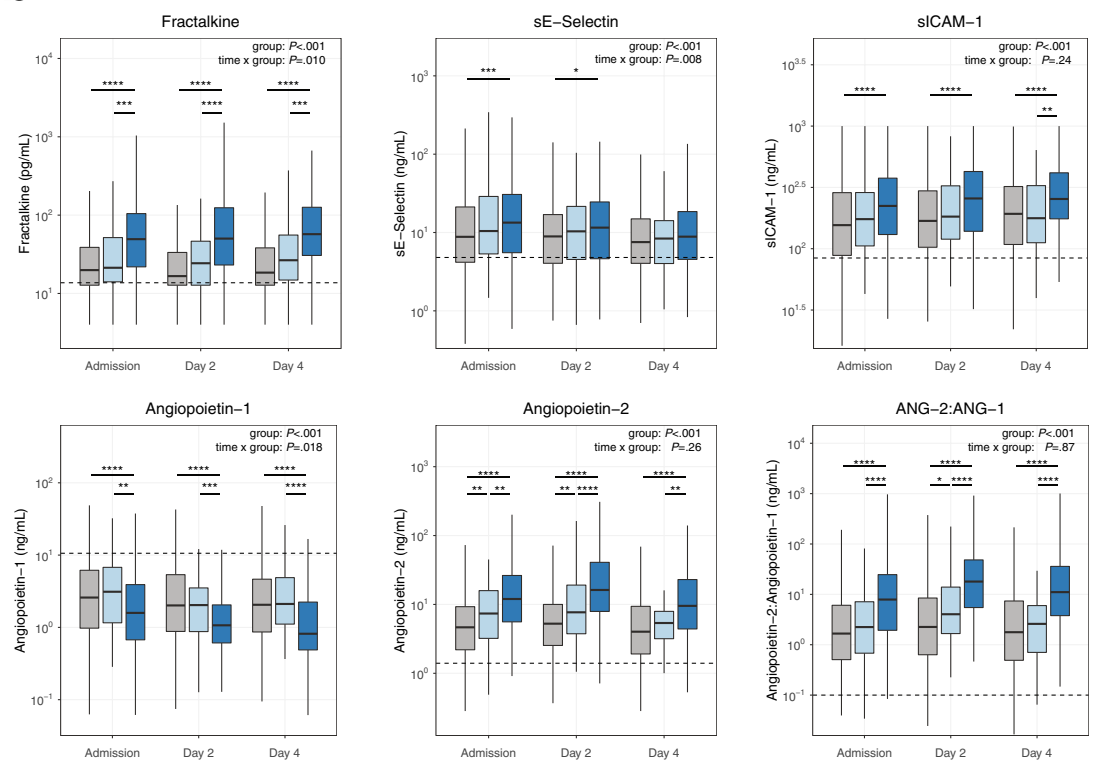

\section{Coagulation activation}
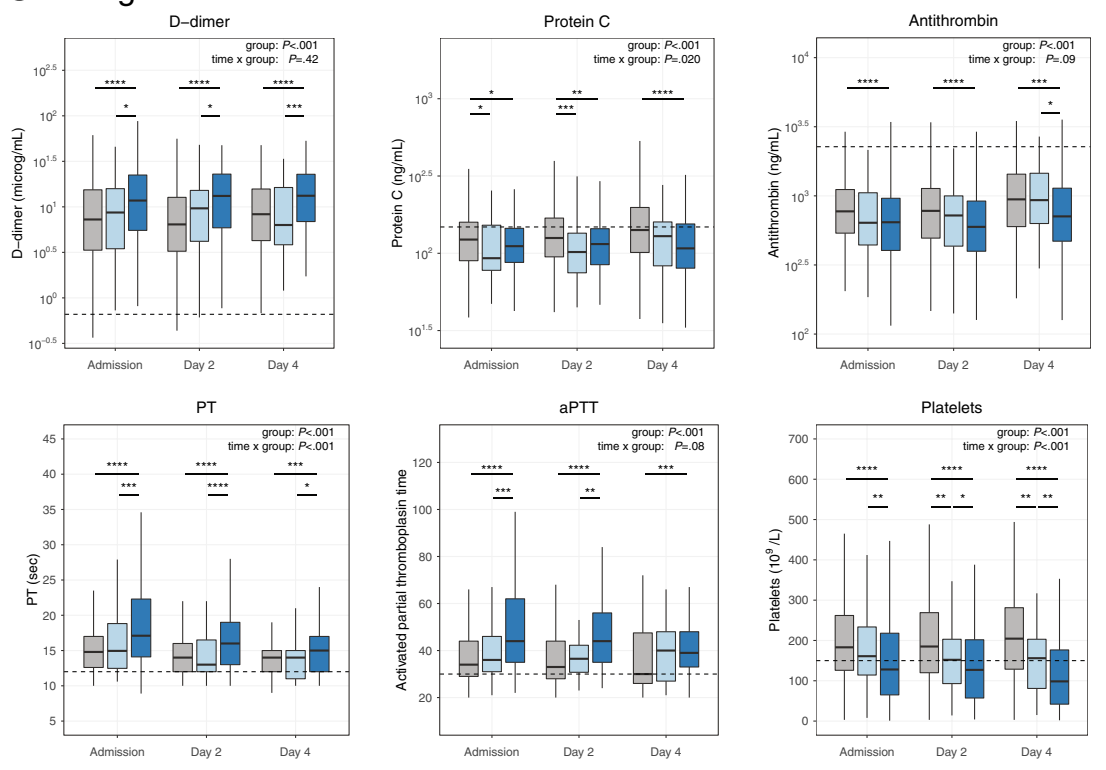

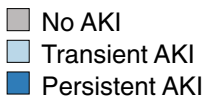

$\square$ No AKI
$\square$ Transient AKI

$\square$ Persistent AKI 
inflammatory responses upon admission. Interestingly, whereas cytokine levels normalized by day 4 in patients with transient AKI, the persistence of AKI was associated with sustained inflammatory responses along the first four days. In addition, patients who went on to experience persistent AKI demonstrated stronger endothelial cell and coagulation activation and a more profound loss of vascular integrity early from admission until day 4 , whereas patients with a transient AKI did not differ from patients without AKI. Although our study does not prove causality, the remaining sustained hyperinflammation and loss of vascular integrity in patients with persistent AKI after matching for severity support a possible role of these host response pathways in the course of AKI during human sepsis. Experimental investigations support the hypothesis implicating inflammation and endothelial dysfunction in the pathogenesis of S-AKI [52-56]. Leukocyte transcriptomic responses showed minimal differences with respect to the presence or persistence of AKI. Notably, this finding does not exclude a role for leukocytes that have migrated into renal tissue, a possibility that is difficult to investigate in large clinical cohorts such as described here. Similar host response abnormalities were also found in patients admitted for noninfectious condition, suggesting that analogous pathophysiological phenomena may be involved in the more general context of critical illness.
Our study has strengths and limitations. This is the first evaluation of the recent ADQI consensus definitions for transient and persistent AKI [14], done in a large, well-characterized cohort of sepsis patients. Daily RIFLE scores were collected prospectively, providing a more accurate estimation of renal function than retrospective severity score calculations [57]. The sequential measurements of plasma biomarkers during the first four ICU days provide valuable insights into the trajectories of the host response to sepsis and renal function. Multiple subgroup and sensitivity analyses strengthened the robustness of our conclusions. Limitations include the retrospective classification of the evolution of AKI, the frequent difficulty to determine the exact timing of organ dysfunction in sepsis patients, which requires to deem ICU-admission the onset of AKI, and the lack of clinical and biological data beyond ICU stay, which precludes adequate assessment of the persistence of AKI beyond day 7, defining acute kidney disease [14]. Although data were collected several years ago (2011-2013), guidelines for the management of AKI and use of renal replacement therapy have not changed since then $[35,58,59]$.

\section{Conclusions}

In patients with sepsis, persistent AKI is independently associated with short- and long-term mortality. Sepsisinduced loss of vascular integrity and sustained inflammation may contribute to the persistence of AKI.

\footnotetext{
(See figure on next page.)

Fig. 3 Leukocyte genomic responses upon admission in sepsis patients without, transient, or persistent acute kidney injury. a Volcano plots illustrating the differences in leukocyte genomic responses (integrating log2 fold changes and multiple-test adjusted probabilities) between sepsis patients without acute kidney injury (AKI) on admission and healthy subjects (left), between patients with transient AKI and healthy subjects (center), and between patients with persistent AKI and healthy subjects (right). Considering adjusted $P<.05,9037,8303$ and 9467 genes were identified as differentially expressed in patients without AKI, patients with transient AKI and patients with persistent AKI on admission vs healthy subjects, respectively. Blue dots represent significantly underexpressed genes (adjusted $P<.05$, fold expression $<-1.5$ ), whereas red dots represent significantly overexpressed genes (adjusted $P<.05$, fold expression $>1.5$ ) in patients relative to healthy controls. Horizontal dotted line indicates multiple-test adjusted Benjamini-Hochberg $(\mathrm{BH}) P<.05$ threshold. Within plots, pie charts show the extent of gene expression changes: Blue slices show significantly underexpressed genes (adjusted $P<.05$ and expression more than 1.5-times decreased compared with healthy controls), red slices show significantly overexpressed genes (adjusted $P<.05$ and expression more than 1.5-time increased compared with healthy controls), and grey slices show significantly different gene expression (adjusted $P<.05$ and expression less than 1.5-time increased or decreased compared with healthy controls). b Venn-Euler representation of differentially expressed genes on admission in sepsis patients without, transient or persistent AKI vs healthy subjects (adjusted $P<.05$ ). Red arrows denote overexpressed genes; blue arrows denote underexpressed genes. c Dot plot depicting the common response (log2 fold changes) of patients without, transient, or persistent AKl as compared with healthy subjects. Rho, Spearman's correlation coefficient. d Volcano plot illustrating the differences in leukocyte genomic responses on admission between patients with transient AKI relative to patients without AKI (left), between patients with persistent AKI relative to patients without AKI (center), and between patients with persistent AKI relative to patients with transient AKI (right). Considering adjusted $P<.05$, no gene was differentially expressed in patients with transient AKI versus no AKI and in patients with persistent AKI vs transient AKI, and 2466 genes were differentially expressed between patients with persistent AKI vs patients without AKI. Within plots, pie charts show the extent of gene expression changes compared to the control group
} 
a

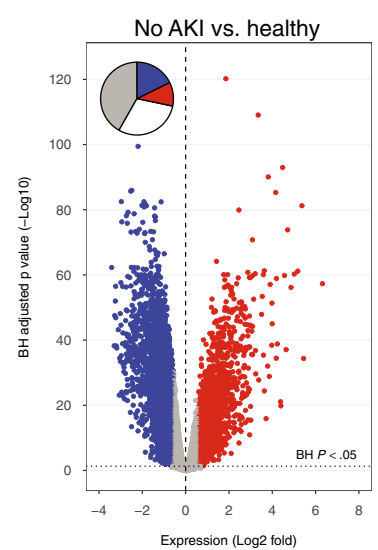

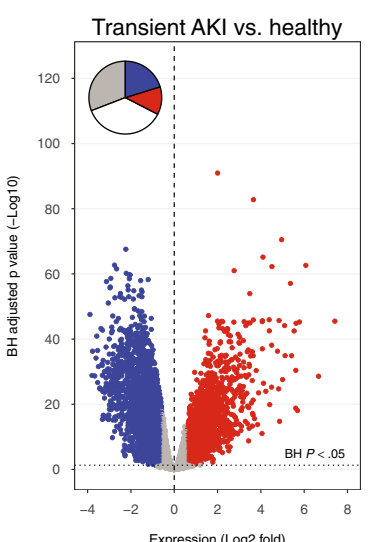

Expression (Log2 fold)

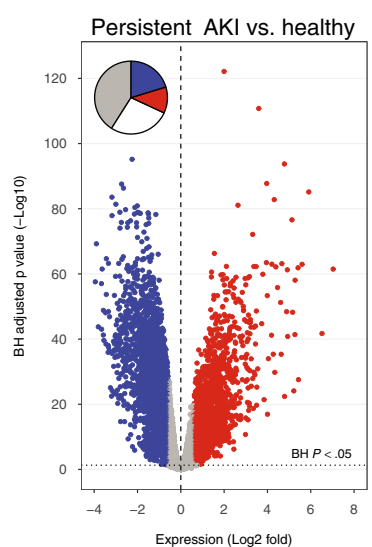

- Underexpressed genes (adj. $p<0.05$, fold expression $<-1.5$ )

- Overexpressed genes (adj. $p<0.05$, fold expression $>1.5$ )

b

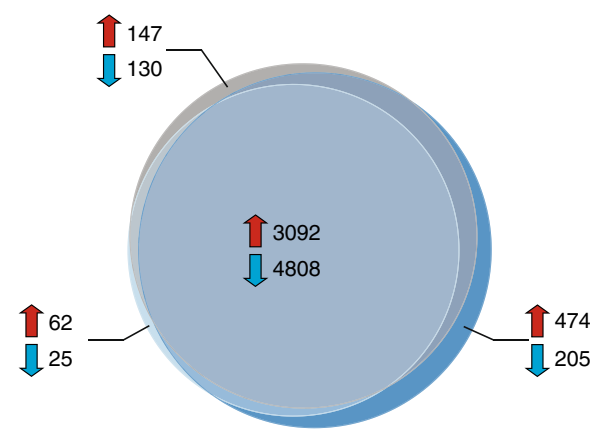

C
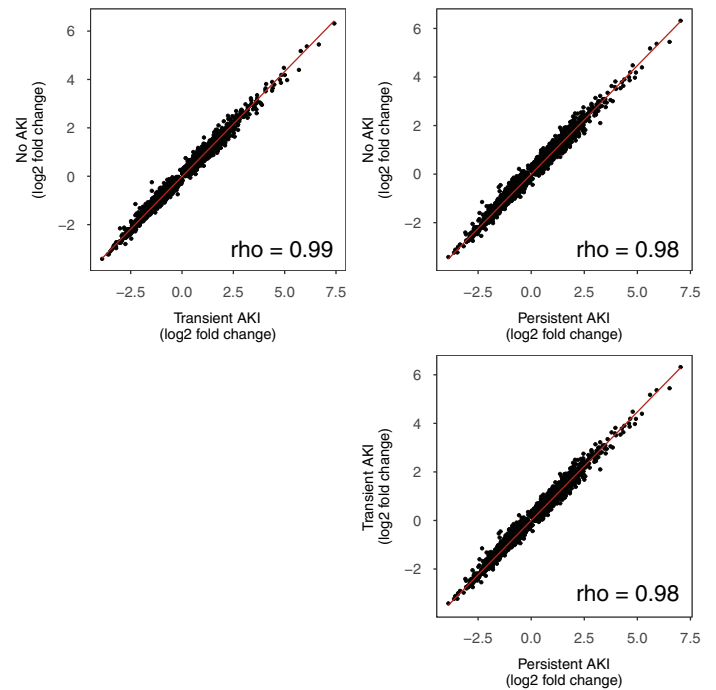

No AKI unique

$\square$ Transient AKI unique

Persistent AKI unique

$\square$ Common response

474

og2 fold change) d

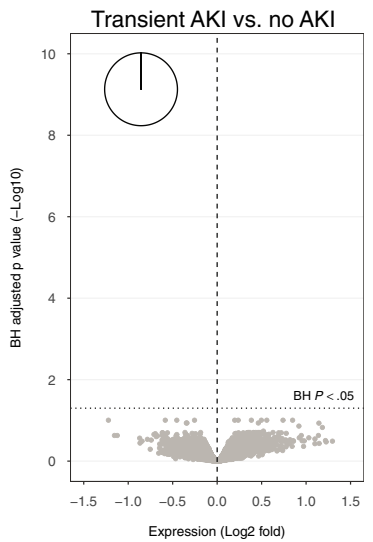

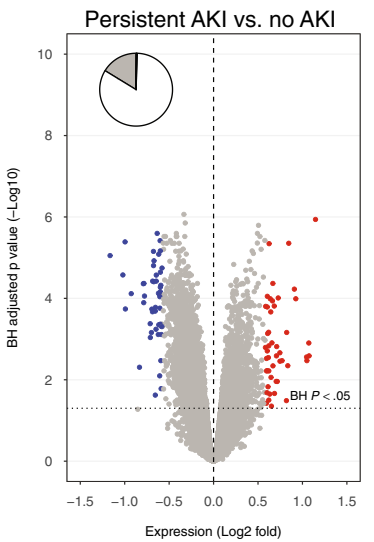

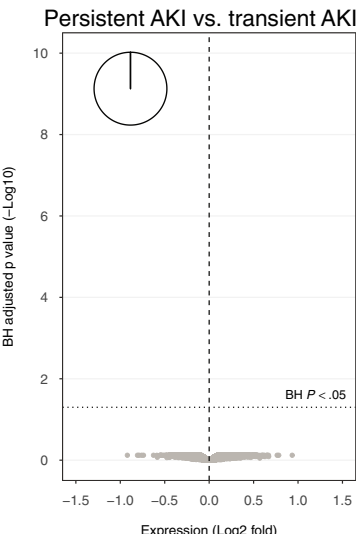

- Underexpressed genes (adj. $p<0.05$, fold expression $<-1.5$ )

- Overexpressed genes (adj. $p<0.05$, fold expression $>1.5$ ) 


\section{Electronic supplementary materia}

The online version of this article (https://doi.org/10.1007/s00134-020-06119-x)

contains supplementary material, which is available to authorized users.

\section{Author details}

${ }^{1}$ Center for Experimental and Molecular Medicine, Amsterdam University Medical Centers, location Academic Medical Center, University of Amsterdam, Room G2-130; Meibergdreef 9, 1105 AZ Amsterdam, The Netherlands. ${ }^{2}$ Department of Clinical Epidemiology, Biostatistics and Bioinformatics, Amsterdam University Medical Centers, location Academic Medical Center, University of Amsterdam, Amsterdam, The Netherlands. ${ }^{3}$ Department of Medical Microbiology, University Medical Center Utrecht, Utrecht, The Netherlands. ${ }^{4}$ Julius Center for Health Sciences and Primary Care, University Medical Center Utrecht, Utrecht, The Netherlands. ${ }^{5}$ Department of Intensive Care Medicine, University Medical Center Utrecht, Utrecht, The Netherlands. ${ }^{6}$ Department of Intensive Care Medicine, and Laboratory of Experimental Intensive Care and Anesthesiology (L.E.I.C.A), Amsterdam University Medical Centers, location Academic Medical Center, University of Amsterdam, Amsterdam, The Netherlands. ${ }^{7}$ Mahidol-Oxford Tropical Medicine Research Unit (MORU), Mahidol University, Bangkok, Thailand. ${ }^{8}$ Nuffield Department of medicine, University of Oxford, Oxford, UK. ${ }^{9}$ Division of Infectious Diseases, Amsterdam University Medical Centers, location Academic Medical Center, University of Amsterdam, Amsterdam, The Netherlands.

\section{Acknowledgements}

The authors acknowledge all members of the Molecular Diagnosis and Risk Stratification of Sepsis consortium for the participation in data collection and especially acknowledge: Friso M. de Beer, M.D., Lieuwe D. J. Bos, Ph.D., Gerie J. Glas, M.D., Roosmarijn T. M. van Hooijdonk, M.D., Ph.D., Janneke Horn, M.D., Ph.D.,Laura R. A. Schouten, M.D., Marleen Straat, M.D., Luuk Wieske, M.D., Ph.D Esther Witteveen, M.D., Department of Intensive Care, Academic Medical Center, University of Amsterdam, The Netherlands; Arie J. Hoogendijk, PhD, Mischa A. Huson, M.D., Maryse A. Wiewel, M.D., Center for Experimental and Molecular Medicine, Amsterdam University Medical Centers, location Academic Medical Center, University of Amsterdam, Amsterdam, The Netherlands, and Center of Infection and Immunity Amsterdam (CINIMA), Amsterdam University Medical Centers, location Academic Medical Center, University of Amsterdam, Amsterdam, The Netherlands; Peter M.C. Klein Klouwenberg, M.D., Ph.D., David S.Y. Ong, M.D., Ph.D., Department of Medical Microbiology, Department of Intensive Care Medicine, and Julius Center for Health Sciences and Primary Care, University Medical Center Utrecht, Utrecht, the Netherlands; Jos F. Frencken, M.D., Maria E. Koster-Brouwer, MSc, Kirsten van de Groep, M.D., Diana M. Verboom, M.D., Department of Intensive Care Medicine and Julius Center for Health Sciences and Primary Care, University Medical Center Utrecht, Utrecht, the Netherlands.

\section{Author's contributions}

FU, HPS, FF, LAvV, and TvdP contributed to the design and conception of the study. All authors contributed to material preparation and to data collection and were involved in the interpretation of the data. FU and BPS did the array analyses. FU had full access to all the data in the study and takes full responsibility for the integrity of the data and the accuracy of the data analysis. FU and TvdP drafted the manuscript, and all authors revised it critically for important intellectual content. All authors gave final approval of this version.

\section{Funding}

This research was performed within the framework of the Center for Translational Molecular Medicine (CTMM) (www.ctmm.nl), project Molecular Diagnosis and Risk Stratification of Sepsis (Grant 04I-201). The sponsor CTMM was not involved in the design and conduction of the study; nor was the sponsor involved in collection, management, analysis, and interpretation of the data or preparation, review or approval of the article. H.P.S. was supported by a grant from the Dutch Kidney Foundation (Kolff Grant Nr. 19OK009). Decision to submit the article was not dependent on the sponsor.

\section{Data availability}

Gene expression datasets are available at the Gene Expression Omnibus public repository of NCBI under accession number GSE65682. Other data generated and/or analyzed during the current study are available on reasonable request.

\section{Compliance with ethical standards}

Conflicts of interest

The authors disclose that they do not have any potential conflicts of interest.

\section{Ethics approval}

Patients were included via an opt-out consent method approved by the institutional review boards of both hospitals (IRB No. 10-056C).

\section{Consent to participate}

Patients were included via an opt-out consent method approved by the institutional review boards of both hospitals (IRB No. 10-056C)

\section{Consent to publish}

Not applicable/all data used for the present study have been anonymized, and the submission does not include information that may identify individual persons.

\section{Open Access}

This article is licensed under a Creative Commons Attribution-NonCommercial 4.0 International License, which permits any non-commercial use, sharing, adaptation, distribution and reproduction in any medium or format, as long as you give appropriate credit to the original author(s) and the source, provide a link to the Creative Commons licence, and indicate if changes were made. The images or other third party material in this article are included in the article's Creative Commons licence, unless indicated otherwise in a credit line to the material. If material is not included in the article's Creative Commons licence and your intended use is not permitted by statutory regulation or exceeds the permitted use, you will need to obtain permission directly from the copyright holder. To view a copy of this licence, visit http://creativecommons.org/licen ses/by-nc/4.0/.

\section{Publisher's Note}

Springer Nature remains neutral with regard to jurisdictional claims in published maps and institutional affiliations.

Received: 14 February 2020 Accepted: 14 May 2020

Published online: 8 June 2020

\section{References}

1. Hoste EAJ, Kellum JA, Selby NM et al (2018) Global epidemiology and outcomes of acute kidney injury. Nat Rev Nephrol 14:607-625. https:// doi.org/10.1038/s41581-018-0052-0

2. Hoste EAJ, Bagshaw SM, Bellomo R et al (2015) Epidemiology of acute kidney injury in critically ill patients: the multinational AKI-EPI study. Intensive Care Med 41:1411-1423. https://doi.org/10.1007/s00134-015-3934-7

3. Gammelager H, Christiansen CF, Johansen MB et al (2014) Three-year risk of cardiovascular disease among intensive care patients with acute kidney injury: a population-based cohort study. Crit Care 18:492. https:// doi.org/10.1186/s13054-014-0492-2

4. Rimes-Stigare C, Frumento P, Bottai M et al (2015) Evolution of chronic renal impairment and long-term mortality after de novo acute kidney injury in the critically ill; a Swedish multi-centre cohort study. Crit Care 19:221. https://doi.org/10.1186/s13054-015-0920-y

5. Uchino S, Kellum JA, Bellomo R et al (2005) Acute renal failure in critically ill patients: a multinational, multicenter study. JAMA 294:813-818. https:// doi.org/10.1001/jama.294.7.813

6. Bagshaw SM, Uchino S, Bellomo R et al (2007) Septic acute kidney injury in critically ill patients: clinical characteristics and outcomes. CJASN 2:431-439. https://doi.org/10.2215/CJN.03681106

7. Peters $\mathrm{E}$, Antonelli M, Wittebole X et al (2018) A worldwide multicentre evaluation of the influence of deterioration or improvement of acute kidney injury on clinical outcome in critically ill patients with and without sepsis at ICU admission: results from The Intensive Care Over Nations audit. Crit Care 22:188. https://doi.org/10.1186/s13054-018-2112-z

8. Bellomo R, Kellum JA, Ronco C et al (2017) Acute kidney injury in sepsis. Intensive Care Med 43:816-828. https://doi.org/10.1007/s0013 4-017-4755-7 
9. Zarbock A, Kellum JA, Schmidt C et al (2016) Effect of early vs delayed initiation of renal replacement therapy on mortality in critically ill patients with acute kidney injury: the ELAIN randomized clinical trial. JAMA 315:2190. https://doi.org/10.1001/jama.2016.5828

10. Gaudry S, Hajage D, Schortgen F et al (2018) Timing of renal support and outcome of septic shock and acute respiratory distress syndrome. a Post Hoc analysis of the AKIKI randomized clinical trial. Am J Respir Crit Care Med 198:58-66. https://doi.org/10.1164/rccm.201706-1255OC

11. Joannidis M, Metnitz B, Bauer P et al (2009) Acute kidney injury in critically ill patients classified by AKIN versus RIFLE using the SAPS 3 database. Intensive Care Med 35:1692-1702. https://doi.org/10.1007/s0013 4-009-1530-4

12. Kellum JA, Chawla LS, Keener C et al (2016) The effects of alternative resuscitation strategies on acute kidney injury in patients with septic shock. Am J Respir Crit Care Med 193:281-287. https://doi.org/10.1164/ rccm.201505-09950C

13. Forni LG, Darmon M, Ostermann M et al (2017) Renal recovery after acute kidney injury. Intensive Care Med 43:855-866. https://doi.org/10.1007/ s00134-017-4809-x

14. Chawla LS, Bellomo R, Bihorac A et al (2017) Acute kidney disease and renal recovery: consensus report of the Acute Disease Quality Initiative (ADQI) 16 Workgroup. Nat Rev Nephrol 13:241-257. https://doi. org/10.1038/nrneph.2017.2

15. Langenberg C, Wan L, Egi M et al (2006) Renal blood flow in experimental septic acute renal failure. Kidney Int 69:1996-2002. https://doi. org/10.1038/sj.ki.5000440

16. Legrand M, Dupuis C, Simon C et al (2013) Association between systemic hemodynamics and septic acute kidney injury in critically ill patients: a retrospective observational study. Crit Care 17:R278. https://doi. org/10.1186/cc13133

17. Langenberg C, Gobe G, Hood S et al (2014) Renal histopathology during experimental septic acute kidney injury and recovery. Crit Care Med 42:e58-e67. https://doi.org/10.1097/CCM.0b013e3182a639da

18. Maiden MJ, Otto S, Brealey JK et al (2016) Structure and function of the kidney in septic shock. a prospective controlled experimental study. Am J Respir Crit Care Med 194:692-700. https://doi.org/10.1164/ rccm.201511-22850C

19. Langenberg C, Bagshaw SM, May CN, Bellomo R (2008) The histopathology of septic acute kidney injury: a systematic review. Crit Care 12:R38. https://doi.org/10.1186/cc6823

20. Lerolle N, Nochy D, Guérot E et al (2010) Histopathology of septic shock induced acute kidney injury: apoptosis and leukocytic infiltration. Intensive Care Med 36:471-478. https://doi.org/10.1007/s00134-009-1723-x

21. Takasu O, Gaut JP, Watanabe E et al (2013) Mechanisms of cardiac and renal dysfunction in patients dying of sepsis. Am J Respir Crit Care Med 187:509-517. https://doi.org/10.1164/rccm.201211-19830C

22. Aslan A, van den Heuvel MC, Stegeman CA et al (2018) Kidney histopathology in lethal human sepsis. Crit Care 22:359. https://doi. org/10.1186/s13054-018-2287-3

23. Parikh CR, Coca SG (2010) Acute kidney injury: defining prerenal azotemia in clinical practice and research. Nat Rev Nephrol 6:641-642. https://doi.org/10.1038/nrneph.2010.128

24. Chawla LS, Seneff MG, Nelson DR et al (2007) Elevated plasma concentrations of IL- 6 and elevated APACHE II score predict acute kidney injury in patients with severe sepsis. CJASN 2:22-30. https://doi. org/10.2215/CJN.02510706

25. Lankadeva YR, Kosaka J, Evans RG et al (2016) Intrarenal and urinary oxygenation during norepinephrine resuscitation in ovine septic acute kidney injury. Kidney Int 90:100-108. https://doi.org/10.1016/j. kint.2016.02.017

26. Gomez H, Ince C, De Backer D et al (2014) A unified theory of sepsisinduced acute kidney injury. Shock 41:3-11. https://doi.org/10.1097/ SHK.0000000000000052

27. (2020) Proceedings of Réanimation 2020, the French Intensive Care Society International Congress. Ann Intensive Care 10:16. https://doi. org/10.1186/s13613-020-0623-7

28. Klein Klouwenberg PMC, Ong DSY, Bos LDJ et al (2013) Interobserver agreement of centers for disease control and prevention criteria for classifying infections in critically ill patients. Crit Care Med 41:23732378. https://doi.org/10.1097/CCM.0b013e3182923712
29. van Vught LA, Klein Klouwenberg PMC, Spitoni C et al (2016) Incidence, risk factors, and attributable mortality of secondary infections in the intensive care unit after admission for sepsis. JAMA 315:1469. https:// doi.org/10.1001/jama.2016.2691

30. Scicluna BP, Klein Klouwenberg PMC, van Vught LA et al (2015) A molecular biomarker to diagnose community-acquired pneumonia on intensive care unit admission. Am J Respir Crit Care Med 192:826-835. https://doi.org/10.1164/rccm.201502-0355OC

31. Scicluna BP, Wiewel MA, van Vught LA et al (2018) Molecular biomarker to assist in diagnosing abdominal sepsis upon ICU admission. Am J Respir Crit Care Med 197:1070-1073. https://doi.org/10.1164/ rCcm.201707-1339LE

32. Meijer MT, Uhel F, Cremer OL et al (2019) Tenascin C plasma levels in critically ill patients with or without sepsis: a multicentre observational study. Shock. https://doi.org/10.1097/SHK.0000000000001481

33. Singer M, Deutschman CS, Seymour CW et al (2016) The third international consensus definitions for sepsis and septic shock (Sepsis-3). JAMA 315:801. https://doi.org/10.1001/jama.2016.0287

34. Bellomo R, Ronco C, Kellum JA et al (2004) Acute renal failure - definition, outcome measures, animal models, fluid therapy and information technology needs: the second international consensus conference of the acute dialysis quality initiative (ADQI) Group. Crit Care 8:R204-9. https://doi.org/10.1186/cc2872

35. KDIGO AKI Work Group (2012) KDIGO clinical practice guideline for acute kidney injury. Kidney Int Suppl 2:1-141. https://doi.org/10.1038/ kisup.2012.7

36. Zeng X, McMahon GM, Brunelli SM et al (2014) Incidence, outcomes, and comparisons across definitions of AKI in hospitalized individuals. CJASN 9:12-20. https://doi.org/10.2215/CJN.02730313

37. Bagshaw SM, Uchino S, Cruz D et al (2009) A comparison of observed versus estimated baseline creatinine for determination of RIFLE class in patients with acute kidney injury. Nephrol Dial Transplant 24:27392744. https://doi.org/10.1093/ndt/gfp159

38. Nejat M, Pickering JW, Walker RJ, Endre ZH (2010) Rapid detection of acute kidney injury by plasma cystatin $\mathrm{C}$ in the intensive care unit. Nephrol Dial Transp 25:3283-3289. https://doi.org/10.1093/ndt/gfa176

39. Bagshaw SM, Bennett M, Haase M et al (2010) Plasma and urine neutrophil gelatinase-associated lipocalin in septic versus non-septic acute kidney injury in critical illness. Intensive Care Med 36:452-461. https:// doi.org/10.1007/s00134-009-1724-9

40. Klein SJ, Brandtner AK, Lehner GF et al (2018) Biomarkers for prediction of renal replacement therapy in acute kidney injury: a systematic review and meta-analysis. Intensive Care Med 44:323-336. https://doi. org/10.1007/s00134-018-5126-8

41. Pierrakos C, Vincent J-L (2010) Sepsis biomarkers: a review. Crit Care 14:R15. https://doi.org/10.1186/cc8872

42. Hotchkiss RS, Moldawer LL, Opal SM et al (2016) Sepsis and septic shock. Nat Rev Dis Primers 2:16045. https://doi.org/10.1038/ nrdp.2016.45

43. Claushuis TAM, van Vught LA, Scicluna BP et al (2016) Thrombocytopenia is associated with a dysregulated host response in critically ill sepsis patients. Blood 127:3062-3072. https://doi.org/10.1182/blood $-2015-11-680744$

44. van der Poll T, van de Veerdonk FL, Scicluna BP, Netea MG (2017) The immunopathology of sepsis and potential therapeutic targets. Nat Rev Immunol 17:407-420. https://doi.org/10.1038/nri.2017.36

45. See EJ, Jayasinghe K, Glassford N et al (2018) Long-term risk of adverse outcomes after acute kidney injury: a systematic review and metaanalysis of cohort studies using consensus definitions of exposure. Kidney Int 95:160-72. https://doi.org/10.1016/j.kint.2018.08.036

46. Chua H-R, Wong W-K, Ong VH et al (2018) Extended mortality and chronic kidney disease after septic acute kidney injury. J Intensive Care Med 11:088506661876461-9. https://doi.org/10.1177/0885066618764617

47. Truche AS, Ragey SP, Souweine B et al (2018) ICU survival and need of renal replacement therapy with respect to AKI duration in critically ill patients. Ann Intensive Care 8:127. https://doi.org/10.1186/s1361 3-018-0467-6

48. Sood MM, Shafer LA, Ho J et al (2014) Early reversible acute kidney injury is associated with improved survival in septic shock. J Crit Care 29:711-717. https://doi.org/10.1016/j.jcrc.2014.04.003 
49. Perinel S, Vincent F, Lautrette A et al (2015) Transient and persistent acute kidney injury and the risk of hospital mortality in critically ill patients. Crit Care Med 43:e269-e275. https://doi.org/10.1097/ CCM. 0000000000001077

50. Kellum JA, Sileanu FE, Bihorac A et al (2017) Recovery after acute kidney injury. Am J Respir Crit Care Med 195:784-791. https://doi. org/10.1164/rccm.201604-07990C

51. Hoste E, Bihorac A, Al-Khafaji A et al (2020) Identification and validation of biomarkers of persistent acute kidney injury: the RUBY study. Intensive Care Med. https://doi.org/10.1007/s00134-019-05919-0

52. Mariano F, Cantaluppi V, Stella M et al (2008) Circulating plasma factors induce tubular and glomerular alterations in septic burns patients. Crit Care 12:R42. https://doi.org/10.1186/cc6848

53. Luo C, Luo F, Zhang L et al (2016) Knockout of interleukin-17A protects against sepsis-associated acute kidney injury. Ann Intensive Care 6:56. https://doi.org/10.1186/s13613-016-0157-1

54. Cunningham PN, Dyanov HM, Park P et al (2002) Acute renal failure in endotoxemia is caused by TNF acting directly on tnf receptor-1 in kidney. J Immunol 168:5817-5823. https://doi.org/10.4049/jimmu nol.168.11.5817
55. Knotek M, Rogachev B, Wang W et al (2001) Endotoxemic renal failure in mice: Role of tumor necrosis factor independent of inducible nitric oxide synthase. Kidney Int 59:2243-2249. https://doi.org/10.104 6/j.1523-1755.2001.00740.x

56. Lelubre C, Vincent J-L (2018) Mechanisms and treatment of organ failure in sepsis. Nat Rev Nephrol 14:417-27. https://doi.org/10.1038/ s41581-018-0005-7

57. Kellum JA, Sileanu FE, Murugan R et al (2015) Classifying AKI by urine output versus serum creatinine level. JASN 26:2231-2238. https://doi. org/10.1681/ASN.2014070724

58. Dellinger RP, Levy MM, Rhodes A et al (2013) Surviving sepsis campaign. Crit Care Med 41:580-637. https://doi.org/10.1097/CCM.0b013e3182 7e83af

59. Rhodes A, Evans LE, Alhazzani W et al (2017) Surviving sepsis campaign: international guidelines for management of sepsis and septic shock: 2016. Intensive Care Med 43:304-377. https://doi.org/10.1007/s0013 4-017-4683-6 